\title{
Spin-Hall effect with quantum group symmetries
}

\author{
Giovanni Landi \\ Dipartimento di Matematica e Informatica, Università di Trieste, \\ Via A. Valerio 12/1, 34127 Trieste \\ and INFN, Sezione di Napoli, Napoli, Italy \\ landi@univ.trieste.it
}

January 10th, 2006

\begin{abstract}
We construct a model of spin-Hall effect on a noncommutative four sphere $S_{\theta}^{4}$ with isospin degrees of freedom, coming from a noncommutative instanton, and invariance under a quantum group $\mathrm{SO}_{\theta}(5)$. The corresponding representation theory allows to explicitly diagonalize the Hamiltonian and construct the ground state; there are both integer and fractional excitations. Similar models exist on higher dimensional spheres $S_{\Theta}^{N}$ and projective spaces $\mathbb{C P}_{\Theta}^{N}$.
\end{abstract}

Dedicated to Rafael Sorkin with friendship and respect.

Key words and phrases: Higher dimensional Hall effects, integer and fractional exitations, quantum groups, noncommutative gauge theories, noncommutative instantons.

MSC: 81V70,58B34,81T13 


\section{Introduction}

The Laughlin wave functions for the fractional quantum Hall effect [16] is not translationally invariant. This problem was overcome in [12 by a model on a sphere with a magnetic monopole at the origin. The full Euclidean group of symmetries of the plane is recovered from the rotation group $\mathrm{SO}(3)$ of symmetries of the sphere. From a more mathematical point of view what one is considering is the Hopf fibration of the sphere $S^{3}$ over the sphere $S^{2}$ with $\mathrm{U}(1)$ as gauge (or structure) group.

The next Hopf fibration of the sphere $S^{7}$ over the sphere $S^{4}$, with $\mathrm{SU}(2)$ as gauge group and $\mathrm{SO}(5)$ as symmetry group, was used in 28] to construct a generalization of the Hall effect with (iso-)spin degrees of freedom coming from a SU(2) Yang monopole [26] (the same as the $\mathrm{SU}(2)$ instanton of [1]). After that, there has been intense theoretical work on generalizations of the Hall effect with the construction of models in higher dimensions and with corresponding internal degrees of freedom (see for instance [14, 10, 3, 13, 4, 22, ).

While is not clear if these models yield theories which are liable to describe the so called spin-Hall effect for which experimental observations have been recently reported [15, 25, they are nonetheless interesting and can be studied on their own. On the one hand they exhibit interesting geometrical structures (see for instance [23, 20]). On the other hand they have been studied in connection with topological field theories, string theory and matrix models (see for instance [11, 6, 29]). In this context methods of noncommutative geometry (or more precisely of fuzzy geometry) are used to construct and analyze these models.

We propose novel models of spin-Hall effect with are invariant under the action of a quantum group. The model that we work out explicitly here is on a noncommutative four sphere $S_{\theta}^{4}$ (or an associated noncommutative plane $\mathbb{R}_{\theta}^{4}$ ), $\theta$ being a deformation parameter, with an $\mathrm{SU}(2)$ noncommutative instanton and invariance under the quantum orthogonal group $\mathrm{SO}_{\theta}(5)$. Orbital symmetries make up a quantum $\mathrm{SO}_{\theta}(4)$. Given the many symmetries, the Hamiltonian describing the excitations of the 'electron gas' is diagonalized explicitly giving both the energies and the eigenstates of the system. There are integer as well as fractional excitations. At the classical value of the deformation parameter, $\theta=0$, we recover fully the model of [28].

As will be reported elsewhere, similar models can be defined on more general noncommutative manifolds, notably spheres $S_{\Theta}^{N}$ (and planes $\mathbb{R}_{\Theta}^{N}$ ) and projective spaces $\mathbb{C P}_{\Theta}^{N}$; here $\Theta$ is a real antisymmetric matrix of deformation parameters. These noncommutative manifolds which are intimately related to the noncommutative tori $\mathbb{T}_{\Theta}^{N}$ were introduced in [9] and have a rich geometrical content; it is worth stressing that they are not fuzzy

spaces. The 2 dimensional noncommutative torus and techniques from noncommutative geometry played a crucial role in the analysis of [2] of the integer quantum Hall effect. For a noncommutative approach to the fractional quantum Hall effect we refer to [19].

\section{Noncommutative spheres}

Toric noncommutative manifolds $M_{\Theta}$ were constructed and studied in [9]. One starts with any (Riemannian spin) manifold $M$ carrying a torus action and then deforms the torus to a noncommutative one governed by a real antisymmetric matrix $\Theta$ of deformation parameters. These noncommutative manifolds were indeed named isospectral deformations 
in that they can be endowed with the structure of a noncommutative Riemannian spin manifold via a spectral triple $\left(C^{\infty}\left(M_{\theta}\right), D, \mathcal{H}\right)$ with the properties of [7]. For this class of examples, the Dirac operator $D$ is the classical one and $\mathcal{H}=L^{2}(M, \mathcal{S})$ is the usual Hilbert space of spinors on which the algebra $C^{\infty}\left(M_{\theta}\right)$ acts in a twisted manner. Thus one twists the algebra and its representation while keeping the geometry unchanged. The resulting noncommutative geometry is isospectral and all spectral properties are preserved including the dimension. Both the algebra and its action on spinors can be given via a "star-type" or "Moyal-type" product. The starting example of [9], the archetype of all these deformations, was a four dimensional sphere $S_{\theta}^{4}$. Noncommutative spheres $S_{\Theta}^{N}$ in any dimensions were made more explicit in [8], and one can also explicitly describe projective spaces $\mathbb{C P}_{\Theta}^{N}$.

In the present paper we shall use the sphere $S_{\theta}^{4}$ and an $\mathrm{SU}(2)$ noncommutative principal fibration over $S_{\theta}^{4}$ constructed in [17]. Similar fibrations can be constructed on higher dimensional noncommutative spheres and also on noncommutative projective spaces.

With $\theta$ a real parameter, the algebra $A\left(S_{\theta}^{4}\right)$ of polynomial functions on the sphere $S_{\theta}^{4}$ is generated by elements $z_{0}=z_{0}^{*}, z_{j}, z_{j}^{*}, j=1,2$, subject to relations

$$
z_{\mu} z_{\nu}=\lambda_{\mu \nu} z_{\nu} z_{\mu}, \quad z_{\mu} z_{\nu}^{*}=\lambda_{\nu \mu} z_{\nu} z_{\mu}^{*}, \quad z_{\mu}^{*} z_{\nu}^{*}=\lambda_{\mu \nu} z_{\nu}^{*} z_{\mu}^{*}, \quad \mu, \nu=0,1,2
$$

with deformation parameters given by

$$
\lambda_{12}=\bar{\lambda}_{21}=: \lambda=e^{2 \pi \mathrm{i} \theta}, \quad \lambda_{j 0}=\lambda_{0 j}=1, \quad j=1,2,
$$

and toghether with the spherical relation $\sum_{\mu} z_{\mu}^{*} z_{\mu}=1$. For $\theta=0$ one recovers the *-algebra of complex polynomial functions on the usual sphere $S^{4}$.

As mentioned above, the sphere $S_{\theta}^{4}$ comes with the structure of a noncommutative Riemannian spin manifold (a spectral triple) $\left(C^{\infty}\left(S_{\theta}^{4}\right), D, \mathcal{H}\right)$ with the undeformed Dirac operator $D$ on $\mathcal{H}=L^{2}\left(S^{4}, \mathcal{S}\right)$, the undeformed Hilbert space of spinors, on which the algebra $C^{\infty}\left(S_{\theta}^{4}\right)$ acts in a twisted manner. The noncommutative geometry being isospectral it follows that $S_{\theta}^{4}$ is four dimensional. On $S_{\theta}^{4}$ there is a compatible exterior calculus: forms are generated by the elements $z_{\mu}, z_{\nu}^{*}$ in degree 0 and elements $\mathrm{d} z_{\mu}, \mathrm{d} z_{\nu}^{*}$ of degree 1 with relations

$$
\begin{gathered}
\mathrm{d} z_{\mu} \mathrm{d} z_{\nu}+\lambda_{\mu \nu} \mathrm{d} z_{\nu} \mathrm{d} z_{\mu}=0, \quad \mathrm{~d} z_{\mu}^{*} \mathrm{~d} z_{\nu}+\lambda_{\nu \mu} \mathrm{d} z_{\nu} \mathrm{d} z_{\mu}^{*}=0, \quad \mathrm{~d} z_{\mu}^{*} \mathrm{~d} z_{\nu}^{*}+\lambda_{\mu \nu} \mathrm{d} z_{\nu}^{*} \mathrm{~d} z_{\mu}^{*}=0, \\
z_{\mu} \mathrm{d} z_{\nu}=\lambda_{\mu \nu} \mathrm{d} z_{\nu} z_{\mu}, \quad z_{\mu}^{*} \mathrm{~d} z_{\nu}=\lambda_{\nu \mu} \mathrm{d} z_{\nu} z_{\mu}^{*}, \quad z_{\mu}^{*} \mathrm{~d} z_{\nu}^{*}=\lambda_{\mu \nu} \mathrm{d} z_{\nu}^{*} z_{\mu}^{*} .
\end{gathered}
$$

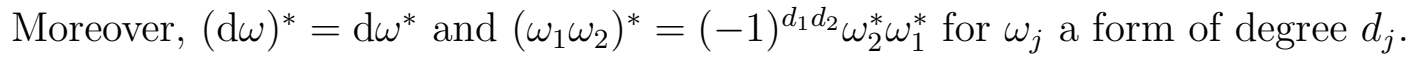

The noncommutative sphere $S_{\theta}^{4}$ can be viewed [8] as the "one-point compactification" of a noncommutative 4-plane $\mathbb{R}_{\theta}^{4}$. With $|\zeta|^{2}=\sum_{j=1}^{2} z_{j}^{*} z_{j}$, the algebra $A\left(S_{\theta}^{4}\right)$ is slightly enlarged by a hermitian central generator $\left(1+|\zeta|^{2}\right)^{-1}$ with relations

$$
\left(1+\sum_{j=1}^{2} z_{j}^{*} z_{j}\right)\left(1+|\zeta|^{2}\right)^{-1}=1, \quad\left(1+|\zeta|^{2}\right)^{-1}\left(1+\sum_{j=1}^{2} z_{j}^{*} z_{j}\right)=1
$$

The elements

$$
\widetilde{z}_{0}=\frac{1-|\zeta|^{2}}{1+|\zeta|^{2}}, \quad \widetilde{z}_{j}=\frac{2 z_{j}}{1+|\zeta|^{2}}, \quad \widetilde{z}_{j}^{*}=\frac{2 z_{j}^{*}}{1+|\zeta|^{2}}
$$


satisfy the same relations as the elements $z_{0}, z_{j}, z_{j}^{*}$. The difference is that the classical point $z_{0}=-1, z_{j}=z_{j}^{*}=0$ of $S_{\theta}^{4}$ is not in the spectrum of $\widetilde{z}_{0}, \widetilde{z}_{j}, \widetilde{z}_{j}^{*}$ and we are describing a noncommutative $\mathbb{R}_{\theta}^{4}$ whose generators can be taken to be $\widetilde{z}_{j}, \widetilde{z}_{j}^{*}, j=1,2$, with relations deduced from (11). In fact, the element $\left(1+|\zeta|^{2}\right)^{-1}$ is smooth and one can deal with the algebra $C^{\infty}\left(\mathbb{R}_{\theta}^{4}\right)$ of smooth functions on $\mathbb{R}_{\theta}^{4}$. One can then cover $S_{\theta}^{4}$ with two "charts" with domain the noncommutative $\mathbb{R}_{\theta}^{4}$ and transitions on $\mathbb{R}_{\theta}^{4} \backslash\{0\}$, the point $z_{j}=z_{j}^{*}=0$ being a classical point of $\mathbb{R}_{\theta}^{4}$.

\section{A noncommutative Hopf fibration}

The sphere $S_{\theta}^{4}$ comes with a noncommutative vector bundles endowed with a self-dual gauge connection [9] which is the generalization of the BPST instanton [1] of SU(2) YangMills theory. This configuration, originally defined on $\mathbb{R}^{4}$, when conformally mapped to $S^{4}$ coincides with the monopole configuration found by Yang [26]. We shall describe at length the noncommutative instanton connection later on since it plays a central role in the spin-Hall effect that we are going to introduce.

Let us start with the $\mathrm{SU}(2)$ noncommutative principal fibration $S_{\theta^{\prime}}^{7} \rightarrow S_{\theta}^{4}$ constructed in [17. With $\lambda_{a b}^{\prime}=e^{2 \pi \mathrm{i} \theta_{a b}^{\prime}}$ and $\left(\theta_{a b}^{\prime}\right)$ a real antisymmetric matrix, the algebra $A\left(S_{\theta^{\prime}}^{7}\right)$ of polynomial functions on the sphere $S_{\theta^{\prime}}^{7}$ is generated by elements $\psi_{a}, \psi_{a}^{*}, a=1, \ldots, 4$, subject to relations

$$
\psi_{a} \psi_{b}=\lambda_{a b} \psi_{b} \psi_{a}, \quad \psi_{a} \psi_{b}^{*}=\lambda_{b a} \psi_{b}^{*} \psi_{a}, \quad \psi_{a}^{*} \psi_{a}^{*}=\lambda_{a b} \psi_{b}^{*} \psi_{a}^{*}
$$

and with the spherical relation $\sum_{a} \psi_{a}^{*} \psi_{a}=1$. At $\theta=0$, it is the $*$-algebra of complex polynomial functions on the sphere $S^{7}$. Again there is a noncommutative geometry via a spectral triple which is isospectral and a compatible exterior calculus. As before, forms are generated by the elements $\psi_{a}, \psi_{b}^{*}$ in degree 0 and elements $\mathrm{d} \psi_{a}, \mathrm{~d} \psi_{b}^{*}$ of degree 1 with relations similar to the ones in (3).

In order to construct the noncommutative Hopf bundle over the given 4-sphere $S_{\theta}^{4}$, we need to select a particular noncommutative 7 dimensional sphere $S_{\theta^{\prime}}^{7}$. We take the one corresponding to the following deformation parameters

$$
\lambda_{a b}^{\prime}=\left(\begin{array}{cccc}
1 & 1 & \bar{\mu} & \mu \\
1 & 1 & \mu & \bar{\mu} \\
\mu & \bar{\mu} & 1 & 1 \\
\bar{\mu} & \mu & 1 & 1
\end{array}\right), \quad \mu=\sqrt{\lambda}, \quad \text { or } \quad \theta_{a b}^{\prime}=\frac{\theta}{2}\left(\begin{array}{cccc}
0 & 0 & -1 & 1 \\
0 & 0 & 1 & -1 \\
1 & -1 & 0 & 0 \\
-1 & 1 & 0 & 0
\end{array}\right) .
$$

The previous choice is essentially the only one that allows the algebra $A\left(S_{\theta^{\prime}}^{7}\right)$ to carry an action of the group $\mathrm{SU}(2)$ by automorphisms and such that the invariant subalgebra coincides with $A\left(S_{\theta}^{4}\right)$. The best way to see this is by means of the matrix-valued function on $A\left(S_{\theta^{\prime}}^{7}\right)$ (we are changing notations with respect to [17])

$$
\Psi=\left(\begin{array}{cc}
\psi_{1} & -\psi_{2}^{*} \\
\psi_{2} & \psi_{1}^{*} \\
\psi_{3} & -\psi_{4}^{*} \\
\psi_{4} & \psi_{3}^{*}
\end{array}\right)
$$


Then, the commutation relations of the algebra $A\left(S_{\theta^{\prime}}^{7}\right)$, with deformation parameter in (6)), gives that $\Psi^{\dagger} \Psi=\mathbb{I}_{2}$. As a consequence, the matrix-valued function $p=\Psi \Psi^{\dagger}$ is a projection, $p^{2}=p=p^{\dagger}$, and its entries rather that functions in $A\left(S_{\theta^{\prime}}^{7}\right)$ are (the generating) elements of $A\left(S_{\theta}^{4}\right)$. Indeed, the right action of $\mathrm{SU}(2)$ on $A\left(S_{\theta^{\prime}}^{7}\right)$ is simply given by

$$
\alpha_{w}(\Psi)=\Psi w, \quad w=\left(\begin{array}{cc}
w_{1} & -\bar{w}_{2} \\
w_{2} & \bar{w}_{1}
\end{array}\right) \in \mathrm{SU}(2),
$$

from which the invariance of the entries of $p$ follows at once: $p_{w}=\alpha_{w}(\Psi) \alpha_{w}(\Psi)^{\dagger}=p$. Explicitly one finds that,

$$
p=\frac{1}{2}\left(\begin{array}{cccc}
1+z_{0} & 0 & z_{1} & -\bar{\mu} z_{2}^{*} \\
0 & 1+z_{0} & z_{2} & \mu z_{1}^{*} \\
z_{1}^{*} & z_{2}^{*} & 1-z_{0} & 0 \\
-\mu z_{2} & \bar{\mu} z_{1} & 0 & 1-z_{0}
\end{array}\right)
$$

with the generators of $A\left(S_{\theta}^{4}\right)$ identified as bilinears in the $\psi, \psi^{*}$ 's and given by

$$
\begin{aligned}
z_{1} & =2\left(\mu \psi_{3}^{*} \psi_{1}+\psi_{2}^{*} \psi_{4}\right), \quad z_{2}=2\left(-\psi_{1}^{*} \psi_{4}+\bar{\mu} \psi_{3}^{*} \psi_{2}\right), \\
z_{0} & =\psi_{1}^{*} \psi_{1}+\psi_{2}^{*} \psi_{2}-\psi_{3}^{*} \psi_{3}-\psi_{4}^{*} \psi_{4} \\
& =2\left(\psi_{1}^{*} \psi_{1}+\psi_{2}^{*} \psi_{2}\right)-1=1-2\left(\psi_{3}^{*} \psi_{3}+\psi_{4}^{*} \psi_{4}\right) .
\end{aligned}
$$

By using the commutation relations of the $\psi$ 's, one straightforwardly computes that $z_{1}^{*} z_{1}+z_{1}^{*} z_{1}+z_{0}^{2}=1$ and the commutation rules $z_{1} z_{2}=\lambda z_{2} z_{1}, z_{1} z_{2}^{*}=\bar{\lambda} z_{2}^{*} z_{1}$, and that $z_{0}$ is central. The relations (10) could be expressed in the form

$$
z_{\mu}=\sum_{a b} \psi_{a}^{*}\left(\Gamma_{\mu}\right)_{a b} \psi_{b}
$$

with $\Gamma_{\mu}$ twisted 4 by 4 Dirac matrices,

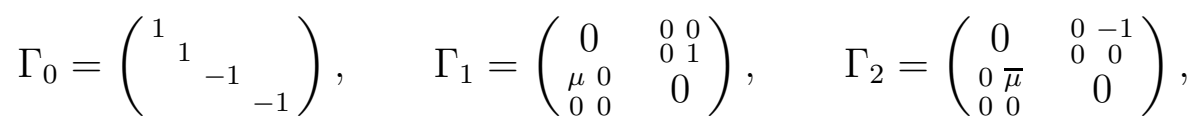

and $\Gamma_{\overline{1}}=\Gamma_{1}^{*}, \Gamma_{\overline{2}}=\Gamma_{2}^{*}$.

The precise sense in which the algebra inclusion $A\left(S_{\theta}^{4}\right) \hookrightarrow A\left(S_{\theta^{\prime}}^{7}\right)$ is a (nontrivial) noncommutative $\mathrm{SU}(2)$ principal bundle is explained in [17]. The projection $p$ determines a noncommutative vector bundle $E$ over $S_{\theta}^{4}$ by giving the collection of its sections (matter fields) as $\Gamma\left(S_{\theta}^{4}, E\right)=p\left[A\left(S_{\theta}^{4}\right)\right]^{4}$ which is technically a right $A\left(S_{\theta}^{4}\right)$-module (i.e. one can multiply a section by a function on the right; not on the left, due to noncommutativity). On this bundle one defines a gauge connection by $\nabla=p \circ \mathrm{d}$ with topological charge equal to -1 and whose curvature $\Omega=p(\mathrm{~d} p)^{2}$ satisfies an anti-selfdual equation $*_{\theta} \Omega=-\Omega$; here the Hodge operator $*_{\theta}$ is defined using the metric of the Dirac operator on $S_{\theta}^{4}$ [8]. With $s u(2)$ the Lie algebra of $\mathrm{SU}(2)$, the $s u(2)$-valued connection 1 form (gauge potential) on $S_{\theta^{\prime}}^{7}$ is most simply written in terms of the matrix-valued function $\Psi$ in (7): it is given by

$$
\omega=\Psi^{\dagger} \mathrm{d} \Psi .
$$

We shall presently "pull it back" to an $s u(2)$-valued gauge potential on $S_{\theta}^{4}$, in fact to "local" expressions on the local chart $\mathbb{R}_{\theta}^{4}$. Classically (i.e. when $\theta=0$ ) one obtains the charge -1 instanton of Yang-Mills gauge theory. The construction of noncommutative instantons will be described at length in [18. 


\section{Gauge fields over $S_{\theta}^{4}$}

We shall first introduce a "local section" of the principal bundle $S_{\theta^{\prime}}^{7} \rightarrow S_{\theta}^{4}$ on the local chart of $S_{\theta}^{4}$ defined in (44). Let $u=\left(u_{1}, u_{2}\right)$ be a complex spinor of modulus one, $u_{1}^{*} u_{1}+$ $u_{2}^{*} u_{2}=1$, and define

$$
\left(\begin{array}{l}
\psi_{1} \\
\psi_{2}
\end{array}\right)=\rho\left(\begin{array}{l}
u_{1} \\
u_{2}
\end{array}\right), \quad\left(\begin{array}{l}
\psi_{3} \\
\psi_{4}
\end{array}\right)=\rho\left(\begin{array}{cc}
z_{1}^{*} & z_{2}^{*} \\
-\mu z_{2} & \bar{\mu} z_{1}
\end{array}\right)\left(\begin{array}{l}
u_{1} \\
u_{2}
\end{array}\right) .
$$

Here $\rho$ is a central element such that $\rho^{2}=\left(1+|\zeta|^{2}\right)^{-1}$, with $|\zeta|^{2}=z_{1}^{*} z_{1}+z_{2}^{*} z_{2}$ as in (4) and the commutations rules of the $u_{j}$ 's with the $z_{k}$ 's are dictated by those of the $\psi_{j}$ :

$$
u_{1} z_{j}=\mu z_{j} u_{1}, \quad u_{2} z_{j}=\bar{\mu} z_{j} u_{2}, \quad j=1,2 .
$$

The right action of $\mathrm{SU}(2)$ rotates the vector $u$ while mapping to the "same point" of $S_{\theta}^{4}$, which, from the choice in (14) is found to be

$$
2\left(\mu \psi_{3}^{*} \psi_{1}+\psi_{2}^{*} \psi_{4}\right)=\widetilde{z}_{1}, \quad 2\left(-\psi_{1}^{*} \psi_{4}+\bar{\mu} \psi_{3}^{*} \psi_{2}\right)=\widetilde{z}_{2}, \quad 2\left(\psi_{1}^{*} \psi_{1}+\psi_{2}^{*} \psi_{2}\right)-1=\widetilde{z}_{0},
$$

and is in the local chart (41), as expected.

By writing the unit vector $u$ as an $\mathrm{SU}(2)$ matrix, $u=\left(\begin{array}{cc}u_{1} & -u_{2}^{*} \\ u_{2} & u_{1}^{*}\end{array}\right)$, and by substituting (14) into (13), we get the gauge potential 1-form on $S_{\theta}^{4}$ as

$$
\Psi^{\dagger} \mathrm{d} \Psi=\eta_{(u)}
$$

with

$$
\begin{aligned}
u \eta_{(u)} u^{*}+u \mathrm{~d} u^{*}=\frac{1}{1+|\zeta|^{2}} & \left(\left(z_{1} \mathrm{~d} z_{1}^{*}-z_{1}^{*} \mathrm{~d} z_{1}-z_{2}^{*} \mathrm{~d} z_{2}+z_{2}^{*} \mathrm{~d} z_{2}\right) \sigma_{3}\right. \\
& \left.+2\left(z_{1} \mathrm{~d} z_{2}^{*}-\bar{\lambda} z_{2}^{*} \mathrm{~d} z_{1}\right) \sigma_{+}+2\left(z_{2} \mathrm{~d} z_{1}^{*}-\lambda z_{1}^{*} \mathrm{~d} z_{2}\right) \sigma_{-}\right) .
\end{aligned}
$$

The corresponding gauge curvature (field strength) is found to be

$$
u F_{(u)} u^{*}=\frac{1}{\left(1+|\zeta|^{2}\right)^{2}}\left(\left(\mathrm{~d} z_{1} \mathrm{~d} z_{1}^{*}-\mathrm{d} z_{2} \mathrm{~d} z_{2}^{*}\right) \sigma_{3}+2\left(\mathrm{~d} z_{1} \mathrm{~d} z_{2}^{*}\right) \sigma_{+}+2\left(\mathrm{~d} z_{2} \mathrm{~d} z_{1}^{*}\right) \sigma_{-}\right) .
$$

Here the (suitably rescaled) Pauli matrices $\sigma_{3}, \sigma_{ \pm}$are the generators of the Lie algebra $s u(2) \simeq \operatorname{sl}(2)$.

On $\mathbb{R}_{\theta}^{4}$ (and $S_{\theta}^{4}$ ) the Hodge operator $*_{\theta}$ acts as "the undeformed one" (remember that we do not deform the Dirac operator, i.e. the metric) and its action is explicitly found to be given by

$$
\begin{array}{ll}
*_{\theta} \mathrm{d} z_{1} \mathrm{~d} z_{2}=\mathrm{d} z_{1} \mathrm{~d} z_{2} & \\
*_{\theta} \mathrm{d} z_{1} \mathrm{~d} z_{1}^{*}=\mathrm{d} z_{2} \mathrm{~d} z_{2}^{*}, & *_{\theta} \mathrm{d} z_{2} \mathrm{~d} z_{2}^{*}=\mathrm{d} z_{1} \mathrm{~d} z_{1}^{*}, \\
*_{\theta} \mathrm{d} z_{1} \mathrm{~d} z_{2}^{*}=-\mathrm{d} z_{1} \mathrm{~d} z_{2}^{*}, & *_{\theta} \mathrm{d} z_{2} \mathrm{~d} z_{1}^{*}=-\mathrm{d} z_{2} \mathrm{~d} z_{1}^{*} .
\end{array}
$$

When acting on (19) we get $*_{\theta}\left(u F_{(u)} u^{*}\right)=-u F_{(u)} u^{*}$, i.e. the curvature is antiself-dual.

As mentioned, when the deformation parameter is set to zero, $\theta=0$, the gauge potential (18) is the BPST instanton [1] of SU(2) Yang-Mills theory. 


\section{$5 \quad$ Quantum groups symmetries}

The noncommutative spheres $S_{\Theta}^{N}$ are quantum homogeneous spaces of quantum orthogonal groups $\mathrm{SO}_{\Theta}(N+1)$, that is there is a coaction of $\mathrm{SO}_{\Theta}(N+1)$ on the functions $A\left(S_{\Theta}^{N}\right)$ leaving the latter coinvariant [24, 8]. Dually, the symmetry can be realized [21] as the action of a universal envelopping algebra $U_{\Theta}(s o(N+1))$. In the present paper we shall describe in details the 4 dimensional case by giving the twisted symmetry action of $U_{\theta}(s o(5))$ on $S_{\theta}^{4}$ which we then lift to $S_{\theta^{\prime}}^{7}$ in a way that the instanton potential decribed above is symmetric under the action.

We recall that the eight roots of the Lie algebra so(5) are two-component vectors $r=\left(r_{1}, r_{2}\right)$ of the form $r=( \pm 1, \pm 1), r=(0, \pm 1), r=( \pm 1,0)$. There are corresponding generators $E_{r}$ of $s o(5)$ toghether with two mutually commuting generators $H_{1}, H_{2}$ of the Cartan subalgebra. The Lie brackets are

$$
\left[H_{1}, H_{2}\right]=0, \quad\left[H_{j}, E_{r}\right]=r_{j} E_{r}, \quad\left[E_{-r}, E_{r}\right]=r_{1} H_{1}+r_{2} H_{2}, \quad\left[E_{r}, E_{r^{\prime}}\right]=N_{r, r^{\prime}} E_{r+r^{\prime}}
$$

with $N_{r, r^{\prime}}=0$ if $r+r^{\prime}$ is not a root. The universal envelopping algebra $U(s o(5))$ is the algebra generated by elements $\left\{H_{j}, E_{r}\right\}$ modulo relations given by the previous Lie brackets $^{1}$. Then, the twisted universal envelopping algebra $U_{\theta}(s o(5))$ is generated as above (i.e. one does not change the algebra structure) but is endowed with a twisted coproduct,

$$
\Delta_{\theta}: U_{\theta}(s o(5)) \rightarrow U_{\theta}(s o(5)) \otimes U_{\theta}(s o(5)),
$$

which, on the generators $E_{r}, H_{j}$, reads

$$
\begin{aligned}
& \Delta_{\theta}\left(E_{r}\right)=E_{r} \otimes \lambda^{-r_{1} H_{2}}+\lambda^{-r_{2} H_{1}} \otimes E_{r}, \\
& \Delta_{\theta}\left(H_{j}\right)=H_{j} \otimes \mathbb{I}+\mathbb{I} \otimes H_{j},
\end{aligned}
$$

with $\lambda=e^{2 \pi \mathrm{i} \theta}$ as before. This coproduct allows to represent $U_{\theta}(s o(5))$ as an algebra of twisted "differential operators" (i.e. derivations of the algebras of functions) on both $S_{\theta}^{4}$ and $S_{\theta^{\prime}}^{7}$ as we shall see below. With counit and antipode given by

$$
\begin{aligned}
& \varepsilon\left(E_{r}\right)=\varepsilon\left(H_{j}\right)=0, \\
& S\left(E_{r}\right)=-\lambda^{r_{2} H_{1}} E_{r} \lambda^{r_{1} H_{2}}, \quad S\left(H_{j}\right)=-H_{j},
\end{aligned}
$$

the algebra $U_{\theta}(s o(5))$ becomes a Hopf algebra [5]. At the classical value of the deformation parameter, $\theta=0$, one recovers the Hopf algebra structure of $U(\operatorname{so}(5))$.

We are ready for the representation of $U_{\theta}(s o(5))$ on $S_{\theta}^{4}$. For convenience, we introduce "partial derivatives", $\partial_{\mu}$ and $\partial_{\mu}^{*}$ with the usual action on the generators of the algebra $A\left(S_{\theta}^{4}\right): \partial_{\mu}\left(z_{\nu}\right)=\delta_{\mu \nu}, \partial_{\mu}\left(z_{\nu}^{*}\right)=0$, and $\partial_{\mu}^{*}\left(z_{\nu}^{*}\right)=\delta_{\mu \nu}, \partial_{\mu}^{*}\left(z_{\nu}\right)=0$. Then, the action of $U_{\theta}(s o(5))$ on $A\left(S_{\theta}^{4}\right)$ is given by the following operators,

$$
\begin{array}{rlrl}
H_{1} & =z_{1} \partial_{1}-z_{1}^{*} \partial_{1}^{*}, & H_{2}=z_{2} \partial_{2}-z_{2}^{*} \partial_{2}^{*}, \\
E_{+1,+1} & =z_{2} \partial_{1}^{*}-z_{1} \partial_{2}^{*}, & E_{+1,-1} & =z_{2}^{*} \partial_{1}^{*}-z_{1} \partial_{2}, \\
E_{+1,0} & =\frac{1}{\sqrt{2}}\left(2 z_{0} \partial_{1}^{*}-z_{1} \partial_{0}\right), & E_{0,+1}=\frac{1}{\sqrt{2}}\left(2 z_{0} \partial_{2}^{*}-z_{2} \partial_{0}\right),
\end{array}
$$

\footnotetext{
${ }^{1}$ There are additional Serre relations; they generate an ideal that needs to be quotiented out. This is not problematic and we shall not dwell upon this point here.
} 
and $E_{-r}=\left(E_{r}\right)^{*}$, with the obvious meaning of the adjoint. These operators (not the partial derivatives!) are extended to the whole of $A\left(S_{\theta}^{4}\right)$ as twisted derivations via the coproduct (23),

$$
\begin{aligned}
& E_{r}(a b)=\Delta_{\theta}\left(E_{r}\right)(a \otimes b)=E_{r}(a) \lambda^{-r_{1} H_{2}}(b)+\lambda^{-r_{2} H_{1}}(a) E_{r}(b), \\
& H_{j}(a b)=\Delta_{\theta}\left(H_{j}\right)(a \otimes b)=H_{j}(a) b+a H_{j}(b)
\end{aligned}
$$

for any two elements $a, b \in A\left(S_{\theta}^{4}\right)$. Accordingly, the partial derivatives $\partial_{\mu}, \partial_{\mu}^{*}$ will obey a twisted Leibniz rule for products of generators. With these twisted rules, one readily checks compatibility with the commutation relations (11) of $A\left(S_{\theta}^{4}\right)$.

The representation of $U_{\theta}(s o(5))$ on $S_{\theta}^{4}$ given in (25) is the fundamental vector representation. When lifted to $S_{\theta^{\prime}}^{7}$ one gets the fundamental spinor representation: as we see from the quadratic relations among corresponding generators, as given in (10), the lifting amounts to take the "square root" representation. The action on $U_{\theta}(s o(5))$ on $A\left(S_{\theta^{\prime}}^{7}\right)$ is constructed by requiring twisted derivation properties via the coproduct (26) so as to reduce to the action (25) on $A\left(S_{\theta}^{4}\right)$ when using the defining quadratic relations (10). The lifted action can be given as the action of twisted matrices $\Gamma$ 's on the $\psi$ 's,

$$
\psi_{a} \mapsto \sum_{b} \Gamma_{a b} \psi_{b}
$$

These matrices $\Gamma$ are the commutators of the twisted Dirac matrices in (12). By writing $\Gamma=\left\{H_{j}, E_{r}\right\}$, a long but straightforward computation shows that in the spinorial representation, they are given explicitly by

$$
\begin{aligned}
& H_{1}=\frac{1}{2}\left(\begin{array}{cccc}
1 & & & \\
& -1 & & \\
& & -1 & \\
& & & 1
\end{array}\right), \quad H_{2}=\frac{1}{2}\left(\begin{array}{cccc}
-1 & & & \\
& 1 & & \\
& & -1 & \\
& & & 1
\end{array}\right),
\end{aligned}
$$

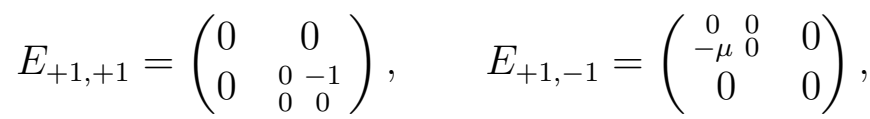

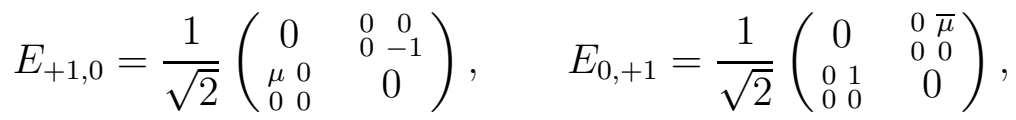

and $E_{-r}=\left(E_{r}\right)^{*}$ as before. As for the $\psi^{*}$ s, one finds instead that they transform as $\psi_{a}^{*} \mapsto \sum_{b}(\widetilde{\Gamma})_{a b} \psi_{b}^{*}$, with

$$
\widetilde{\Gamma}=\sigma \Gamma \sigma^{-1}, \quad \sigma=\left(\begin{array}{ccc}
0-1 & 0 \\
1 & 0 & 0 \\
0 & 1 & 0
\end{array}\right)
$$

at $\mu=1$ (or $\theta=0$ ), one gets $\widetilde{\Gamma}=-\Gamma^{*}$. With the twisted rules (26) for the action on products, one checks compatibility of the above action with the commutation relations (15) of $A\left(S_{\theta^{\prime}}^{7}\right)$. Notice that the $\psi$ 's and the $\psi^{*}$ 's are not mixed by the action and each group transforms into itself. It takes a little algebra to check that the instanton configuration, given in (13) or (18) is invariant under the action of $U_{\theta}(s o(5))$ in (27) or (25).

In fact, the polynomial algebra $A\left(S_{\theta^{\prime}}^{7}\right)$ contains ALL representations of $U_{\theta}(s o(5))$ while the polynomial subalgebra $A\left(S_{\theta}^{4}\right)$ contains only the vector representations. In a sense, $S_{\theta^{\prime}}^{7}$ carries an action of $U_{\theta}(\operatorname{spin}(5))$ and dually a coaction of $\operatorname{Spin}_{\theta}(5)$, a fact that parallels the classical action of $\operatorname{Spin}(5)$ on $S^{7} \simeq \operatorname{Spin}(5) / \mathrm{SU}(2)$. 
From general considerations [5], the representation theory of $U_{\theta}(s o(5))$ is the same as the one of the undeformed counterpart $U(s o(5))$, at least when $\mu$ is not a root of unity; for the latter case there may be additional representations.

There are two fundamental weights $W^{1}=\frac{1}{2}(1,1)$ and $W^{2}=(1,0)$ which correspond to the fundamental spinor representation (27) and vector representation (25) respectively. Each representation is labelled by two integers $s, n$, being characterized by a highest weight $W=s W^{1}+n W^{2}$ and has dimension

$$
d(s, n)=(1+s)(1+n)\left(1+\frac{s+n}{2}\right)\left(1+\frac{s+2 n}{3}\right) .
$$

The integer $s$ measures the "spinorial content" of the representation. A more usual (iso)spin label $I$, such that $2 I=s$, takes integer and half integer values and will be used later on. Since the Lie algebra structure is not changed, the quadratic Casimir operator is

$$
C=H_{1}^{2}+H_{2}^{2}+\sum_{r^{+}}\left(E_{r} E_{-r}+E_{-r} E_{r}\right)
$$

with the sum over the positive roots. This operator is a multiple of the identity in each representation, the factor being given by $C(s, n)=\frac{1}{2}\left(s^{2}+2 n^{2}+2 s n\right)+2 s+3 n$.

\section{The spin-Hall system on $S_{\theta}^{4}$}

The model that we propose will parallel the one constructed in 28] on the classical sphere $S^{4}$. The Hamiltonian of a "single particle" moving on the sphere $S_{\theta}^{4}$ and coupled to the gauge field $\eta=\eta_{(u=1)}$ in (18) is given by

$$
H_{\eta}=-(\mathrm{d}+\eta)^{*}(\mathrm{~d}+\eta)
$$

with all physical constants being set to 1 . We take the gauge potential $\eta$ in an arbitrary representation $I$ of the $s u(2)$ algebra generated by the Pauli matrices $\sigma_{3}, \sigma_{ \pm}$. The isospin label $I$ takes integer and half integer values and the dimension of this representation is $2 I+1$ with corresponding Casimir operator $C_{s u(2)}$ taking the value $I(I+1)$.

We can expand the covariant derivative, $D=\mathrm{d}+\eta$, on the basis of one forms $\mathrm{d} z_{\mu}, \mathrm{d} z_{\mu}^{*}$ as $D=\mathrm{d} z_{\mu} D_{\mu}+\mathrm{d} z_{\mu}^{*} D_{\mu}^{*}$. Then, the Hamiltonian (31) takes the form,

$$
H_{\eta}=\widetilde{H}_{1}^{2}+\widetilde{H}_{2}^{2}+\sum_{r^{+}}\left(\widetilde{E}_{r} \widetilde{E}_{-r}+\widetilde{E}_{-r} \widetilde{E}_{r}\right)
$$

where the operators $\widetilde{H}_{j}$ and $\widetilde{E}_{r}$ are the same as in (25) with the partial derivatives $\partial_{\mu}$, $\partial_{\mu}^{*}$ substituted by the covariant ones $D_{\mu}, D_{\mu}^{*}$ and the sum is over positive roots.

Without the gauge potential the Hamiltonian $H_{\eta=0}$ is just the Casimir operator (30) of the twisted algebra $U_{\theta}(s o(5))$. In a non vanishing gauge field, one needs also the curvature $F=F_{(u=1)}$ of the connection. We shall then also expand the curvature $F$ given in (19) as a two form

$$
F=F_{00} \mathrm{~d} z_{0} \mathrm{~d} z_{0}+\frac{1}{2} F_{\varepsilon_{\mu} \mu, \varepsilon_{\nu} \nu} \mathrm{d} z_{\varepsilon_{\mu} \mu} \mathrm{d} z_{\varepsilon_{\nu} \nu}
$$


with $\varepsilon_{\mu}$ and $\varepsilon_{\nu}$ taking values \pm 1 (remember the root structure of $s o(5)$ ) and with the convention that $\mathrm{d} z_{-\mu}=\mathrm{d} z_{\mu}^{*}$. Some algebra shows that the operators

$$
H_{1}=\widetilde{H}_{1}-F_{00}, \quad H_{2}=\widetilde{H}_{2}-F_{00}, \quad E_{\varepsilon_{\mu} \mu, \varepsilon_{\nu} \nu}=\widetilde{E}_{\varepsilon_{\mu} \mu, \varepsilon_{\nu} \nu}-F_{\varepsilon_{\mu} \mu, \varepsilon_{\nu} \nu}
$$

close the commutation relations (21) of the Lie algebra $s o(5)$; the analogous classical result was established in [27]. Now, the operators $F_{\varepsilon_{\mu} \mu, \varepsilon_{\nu} \nu}$ carry an isospin representation labelled by $I$. With this, one finds that the Hamiltonian (32) is given in terms of the Casimir operators $C$ and $C_{s u(2)}$ (the latter coming from the instanton field strength) as

$$
H_{\eta}=C-2 C_{s u(2)}
$$

The eigenvalues of this Hamiltonian are then given by the energies

$$
\begin{aligned}
E(I, n) & =C(s=2 I, n)-2 I(I+1) \\
& =n^{2}+n(2 I+3)+2 I
\end{aligned}
$$

with degeneracy $d(s=2 I, n)$. The integer $n$ labels Landau levels and $I$, which plays the role of the magnetic flux, label the degeneracy in each Landau level.

The ground state for a given $I$ is obtained when $n=0$; it has energy

$$
E_{0}(I)=2 I
$$

and its degeneration is

$$
d_{0}(I)=d(s=2 I, n=0)=\frac{1}{6}(1+2 I)(2+2 I)(3+2 I) .
$$

States with $n>0$ are separated from the ground state by a finite gap. Using the representation theory of $U_{\theta}(s o(5))$ constructed above, it is straightforward to write down the wave functions of the ground state. The spinor $\psi=\left(\psi_{1}, \ldots, \psi_{4}\right)$ is an eigenfunction of the Hamiltonian (32) with $I=\frac{1}{2}$, the fundamental spinorial representation, and from (27) and (28) we see that the highest weight vector of this representation is $\psi_{4}$ :

$$
H_{1}\left(\psi_{4}\right)=\frac{1}{2}=H_{2}\left(\psi_{4}\right)
$$

Thus, a basis for the ground state, with is just the representation with $s=2 I$ and $n=0$, can be obtained by the corresponding highest weight vector, that is $\Phi=\left(\psi_{4}\right)^{2 I}$, by repeated action of the operators $E_{r}$ as twisted derivations. The results are $d_{0}(I)$ ( $\mu$-symmetric) polynomials of degree $2 I$ in $\psi_{1}, \ldots, \psi_{4}$.

With the local section (14), the ground state with "orbital coordinates" $\vec{z}=z_{\mu}$ and "isospin coordinates" $\vec{I}=\left(I_{+}, I_{-}, I_{3}\right)=\left(\sigma_{+}, \sigma_{-}, \sigma_{3}\right)$ is given by the wave functions,

$$
\Phi\left(\vec{z}, \vec{I} ; k_{1}, k_{2}, k_{3}, k_{4}\right)=\operatorname{Sym}_{\mu}\left(\left(\psi_{1}\right)^{k_{1}}\left(\psi_{2}\right)^{k_{2}}\left(\psi_{3}\right)^{k_{3}}\left(\psi_{4}\right)^{k_{4}}\right)
$$

with integer $k_{1}+k_{2}+k_{3}+k_{4}=2 I$, and $S y m_{\mu}$ denotes a (tensor product) symmetrization which takes into account factors of the deformation parameter due to the commutation relations (5) of the $\psi$ 's. As said, the action of $U_{\theta}(s o(5))$ on (40) is constructed out of the 
fundamental spinorial representation (27) with a repeated use of the twisted coaction (26). The previous are exact eigenstates of the Hamiltonian corresponding to the eigenvalue $E=2 I$ with $d_{0}(I)$ degeneracy.

There are also fractional excitations: they are obtained from the highest weight vector $\Phi_{q}=(\Phi)^{q}=\left(\psi_{4}\right)^{2 I q}$, with odd integer $q$. The corresponding wave functions are fermionic eigenfunctions of the Hamiltonian in the lowest Landau level and are indeed $\mu$-symmetric homogeneous polynomials of degree $2 I q$ obtained, as before by repeated action of the operators $E_{r}$ as twisted derivations. The degeneracy is now $d_{0}(q I)$ and the filling factor of the lowest Landau level is fractional,

$$
\nu=d_{0}(I) / d_{0}(q I) \sim q^{-3}+O(1 / I),
$$

The large isospin limit $I \rightarrow \infty$ should be taken with care in order for the energy to remain finite 28 . One needs also to take $R \rightarrow \infty$ where $R$ is the "radius" of the sphere $S_{\theta}^{4}$. By inserting back physical quantities, the energy eigenvalues are

$$
E(I, n)=\frac{\hbar^{2}}{2 m R^{2}}\left(n^{2}+n(2 I+3)+2 I\right)
$$

The limit is taken in such a manner that the "magnetic length" $\ell_{0}=\sqrt{2 I / R^{2}}$ is constant. In this limit, with a finite Landau level $n$, the energy is

$$
E(I, n) \sim \frac{\hbar^{2} l_{0}^{2}}{2 m}(n+1) .
$$

There are also "orbital" $\mathrm{SO}_{\theta}(4)$ symmetries; these are generated by the elements

$$
L_{a}^{+}=\left(\frac{1}{2}\left(H_{1}+H_{2}\right), E_{+1,+1}, E_{-1,-1}\right), \quad L_{a}^{-}=\left(\frac{1}{2}\left(H_{1}-H_{2}\right), E_{+1,-1}, E_{-1,+1}\right),
$$

with the label $a \in\{3,+,-\}$. One checks that the two sets $L_{a}^{ \pm}$close the $s u(2)$ Lie algebra, having $s o(4)=s u(2) \oplus s u(2)$. The presence of the instanton gauge potential will modify these generators to $\widetilde{L}_{a}^{+}=L_{a}^{+}$and $\widetilde{L}_{a}^{-}=L_{a}^{-}+\sigma_{a}$ due to the commutation relations of the generators $\sigma_{a}$ which are here in a generic representation with isospin $I$.

The representations of the corresponding envelopping algebra $U_{\theta}(s o(4))$ are determined by two spin labels $\left(l^{+}, l^{-}\right)$both taking integer and half integer values. It is straigthforward to check that the pair $\left(\psi_{1}, \psi_{2}\right)$ trasforms according to the representation $\left(0, \frac{1}{2}\right)$ while the the pair $\left(\psi_{4}, \psi_{3}\right)$ trasforms according to the representation $\left(\frac{1}{2}, 0\right)$. Then, the ground states wave functions (40) are in the representation

$$
l^{+}=\frac{1}{2}\left(k_{3}+k_{4}\right), \quad l^{-}=\frac{1}{2}\left(k_{1}+k_{2}\right) .
$$

Furthermore, they are also eigenstates of the operators $\widetilde{L}_{3}^{+}$and $\widetilde{L}_{3}^{-}$with eigenvalues given by $l_{3}^{+}=k_{4}-k_{3}$ and $l_{3}^{-}=k_{1}-k_{2}$ respectively.

\section{$7 \quad$ Final remarks}

We have presented a gauge field theory on a noncommutative four sphere which gives explicit results. The gauge fields provides iso-spin degrees of freedom and there are 
symmetries under a quantum orthogonal group $\mathrm{SO}_{\theta}(5)$. The symmetries are enough to explicit diagonalize the Hamiltonian of excitations moving on the sphere $S_{\theta}^{4}$ in the field of a noncommutative instanton. For the many body problem, there are both integer and half integer modes. The model is a generalization to noncommutative spaces of generalizations of spin-Hall models.

There are several open problems and questions starting with the physical meaning of the noncommutativity parameter. In the usual quantum Hall effect the filling factor plays the role of the deformation parameter and is directly accesible to experiments. In fact, the deformation parameter could be seen as the inverse of the magnetic field in which the carriers move. One can expect a similar interpretation for the model presented here. Also, one needs an analysis of the dependence on the noncommutativity parameter $\mu$ of the ground states wave functions as well as of the quantum many body problem and of edge states. Finally, there are potential applications to string theories and matrix models.

\section{Acknowledgments}

I am grateful to K. Hasebe for providing me with a copy of the paper 28] and for useful remarks. W. van Suijlekom and J. Várilly have made a number suggestions. Finally, I thank the referees for helpful comments. 


\section{References}

[1] A. Belavin, A. Polyakov, A. Schwarz, Y. Tyupkin, Pseudoparticle Solutions of the Yang-Mills Equations, Phys. Lett. 59B (1975) 85-87.

[2] J. Bellissard, A. van Elst, H. Schulz-Baldes, The noncommutative geometry of the quantum Hall effect, J. Math. Phys. 35 (1994) 5373-5451.

[3] B.A. Bernevig, J.-P. Hu, N. Toumbas, S.-C. Zhang, The Eight Dimensional Quantum Hall Effect and the Octonions, Phys. Rev. Lett. 91 (2003) 236803.

[4] B. A. Bernevig, S-C. Zhang, Quantum Spin Hall Effect, arXiv: cond-mat/0504147.

[5] V. Chari, A. Pressley, A guide to quantum groups, Cambridge University Press, Cambridge, 1994.

[6] Y.-X. Chen, Matrix models of 4-dimensional quantum Hall fluids, arXiv:hep-th/0209182.

[7] A. Connes, Gravity coupled with matter and the foundation of noncommutative geometry, Commun. Math. Phys., 182 (1996) 155-176.

[8] A. Connes, M. Dubois-Violette, Noncommutative finite-dimensional manifolds. I. Spherical manifolds and related examples, Commun. Math. Phys. 230 (2002) 539579.

[9] A. Connes, G. Landi, Noncommutative manifolds: The instanton algebra and isospectral deformations, Commun. Math. Phys. 221 (2001) 141-159.

[10] H. Elvang, J. Polchinski, The Quantum Hall Effect on $R^{4}$, arXiv:hep-th/0209104.

[11] M. Fabinger, Higher-Dimensional Quantum Hall Effect in String Theory, JHEP 0205 (2002) 037.

[12] F.D. Haldane, Fractional quantization of the Hall effect: A hierarchy of incompressible quantum fluid states, Phys. Rev. Lett. 51 (1983) 605-608.

[13] K. Hasebe, Y. Kimura, Dimensional Hierarchy in Quantum Hall Effects on Fuzzy Spheres, Phys. Lett. B602 (2004) 255-260.

[14] D. Karabali, V.P. Nair, Quantum Hall Effect in Higher Dimensions, Nucl. Phys. B641 (2002) 533-546.

[15] Y. K. Kato, R. C. Myers, A. C. Gossard, D.D. Awschalom, Observation of the spin Hall effect in semiconductors, Science, 306 (2004) 1910.

[16] R.B. Laughlin, Anomalous quantum Hall effect: An incompressible quantum fluid with fractionally charged excitations, Phys. Rev. Lett. 50 (1983) 1395-1398.

[17] G. Landi, W. van Suijlekom, Principal fibrations from noncommutative spheres, Commun. Math. Phys. 260 (2005) 203-225. 
[18] G. Landi, W. van Suijlekom, Noncommutative instantons from twisted conformal symmetries, work in progress.

[19] M. Marcolli, V. Mathai, Toward the fractional quantum Hall effect: A noncommutative geometry perspective, arXiv: cond-mat/0502356.

[20] G. Meng, Geometric construction of the Quantum Hall Effect in all even dimensions, J. Phys. A36 (2003) 9415-9424.

[21] A. Sitarz, Twists and spectral triples for isospectral deformations, Lett. Math. Phys. 58 (2001) 69-79.

[22] S. Murakami, Intrinsic Spin Hall Effect, arXiv: cond-mat/0504353.

[23] G. Sparling, Twistor theory and the four dimensional quantum Hall effect of Zhang and $\mathrm{Hu}$, arXiv: cond-mat/0211679.

[24] J.C. Várilly, Quantum symmetry groups of noncommutative spheres, Commun. Math. Phys. 221 (2001) 511-523.

[25] J. Wunderlich, B. Kaestner, J. Sinova, T. Jungwirth, Experimental observation of the spin-Hall effect in a two dimensional spin-orbit coupled semiconductor system, arXiv: cond-mat/0410295.

[26] C.N. Yang, Generalization of Dirac's monopole to $S U_{2}$ gauge fields, J. Math. Phys. 19 (1978) 320-328.

[27] C.N. Yang, $S U_{2}$ monopole harmonics, J. Math. Phys. 19 (1978) 2622-2627.

[28] S.-C. Zhang, J.-P. Hu A four-dimensional generalization of the quantum Hall effect, Science 294 (2001) 823; and arXiv: cond-mat/0110572.

[29] S.-C. Zhang, To see the word in a grain of sand, arXiv:hep-th/0210162. 